\title{
The study on analysis method of collision mechanics between motorcycles and automobiles
}

\author{
Ming Ni \\ Department of Physics and Technology, Kunming University, Kunming 650214, China \\ kmxynm@163.com
}

\begin{abstract}
Keywords: Collision mechanics; Dynamics; The deformation energy; Energy conservation; Analysis method
\end{abstract}

\begin{abstract}
In the accident reconstruction, the measurement is often performed according to the accidents information left in the scene of the accident, such as collision point, braking traces, scattered, fallout, the throw distance of the body, the throw distance of the two-wheeler, etc. With the aid of these effective information to reconstruct the accident process, the theoretical basis for the decision on the responsibility for accident can be provided. However, whether measured parameters of the scene of the accident or experience parameters (such as the adhesion coefficient) contain uncertainty factors, whose result is a range. Therefore, in order to ensure the objectivity of the accident appraisal results, the uncertainty analysis theory is introduced to make the results more credibility.
\end{abstract}

\section{Introduction}

The collision accident between a motorcycle and an automobile is more complex than that between a bicycle and an automobile. Three collision processes can be divided between a motorcycle and an automobile[1]:

The process before the collision: the process which is from the car drivers' finding the emergency to two vehicles' contact.

Direct collision process: the process from the contact of the two vehicles to their separation.

The process after the collision: the process two vehicles' separation to their full stop.

The calculation model before and after collision is the trajectory model, and the direct collision is collision model. the forward simulation method, while the collision model calculation conducted with the model which is after the collision is called the backstepping method. At present, the backstepping method is not only applied to solve the collision speed, but also analyzed to obtain the initial conditions [1]. There are some software based on the backstepping method such as PC CRASH [2] SMASH [3], CM accident analysis software of Tsinghua University [4] in the acciden identificationt. Those software are based on the two-dimensional collision model, largely refer to momentum model (momentum conservation theorem, the law of conservation of angular momentum) and deformation experience formula. The recostruction trajectory model of the traffic accident determines the computing power and precision before and after collision, which mainly include the vehicle kinematic model, the dynamic model and the tire model, etc. [5]. The applicability of the kinematics and dynamics model of vehicle has an important effect on the trajectory model, and there are differences on the calculation accuracy. The 15-degree freedom HVOSM three-dimensional vehicle dynamics model developed by Calspan corporation [6] (road vehicle simulation model) and the 71-degree freedom of PHASE4 / EDVDS three-dimensional vehicle dynamics model developed by University of Michigan Transportation Research Institute [7] apply to commercial vehicle dynamics simulation. SIMON three-dimensional vehicle dynamics model developed by ED company uses the semi-empirical tire model of HSRI, realizing multiple vehicle collision simulation [8].

\section{The overall accident analysis}

Accident forms can be divided into three categories. the automobile and themotorcycle , the car and the electric bicycle, the automobile and the bicycle. The overall statistics is conducted from six 
aspects to show the collision form, the total number of accidents, casualties and fatality rate. The accident overall situation is shown in table 1. Among them, the fatality rate refers to the ratio of the total number of deaths and injuries, namely:

The fatality rate $=$ the number of deaths/ the total number of injuries

Table 1 the total situation of accidents

\begin{tabular}{|c|c|c|c|c|c|c|c|c|c|}
\hline the accident \\
forms
\end{tabular}

Note: the unit of the number of deaths injuries is person, and other statistics unit is:case

Seen from table 1, in the three different forms of 212 accidents, the total number of casualties is 262 and the fatality rate is 0.56 . Among them, the number of accidents between and motorcycle is the largest , accounting for $51.89 \%$ of total accidents, while the number of accidents between the automobile and the bicycle is the smallest, accounting for $11.32 \%$ of the total accident. But from the severity of injuries, the most serious accidents are between the automobile and the bicycle, whose fatality rate is 0.81 . Followed by the accidents the automobile and the electric bicycle, with the fatality rate of 0.55 .

\section{The collision of deformation energy method}

The wheel base of the motorcycle almost don't deform when the speed of the motorcycle crashing the automobile is less than $25 \mathrm{~km} / \mathrm{h}$; when its collision speed is more than $30 \sim 40$, the wheel base of the motorcycle deformation increases sharply [9]. Ignoring the noise produced by deformation in the process of the automobile collision, heat energy loss and vehicle rotational kinetic energy, and assuming there is the common speed on the contact surfaces at the end of the collision, Campbell studied the relationshipbetween the permanent deformation of the vehicles and collision speed [10] :

$$
V_{c}=b_{0}+b_{1} C
$$

Among them, $\mathrm{C}$ is the permanent deformation; $\mathrm{Vc}$ is the collision speed; $b_{0}$ is the deformation speed under zero; $b_{1}$ is the slope obtained by the test on vehicle collision with fixed obstacles .Campbell have further studied the force of the units damaged width in the collision area:

$$
F / W=A+B C
$$

Among them, the $\mathrm{F} / \mathrm{W}$ is the collision force on the damaged width of the unit, as shown in figure 1; $\mathrm{A}$ is the collision force of the unit width under the zero deformation; $\mathrm{A}$ and $\mathrm{B}$ can be referred to as the stiffness coefficient. 


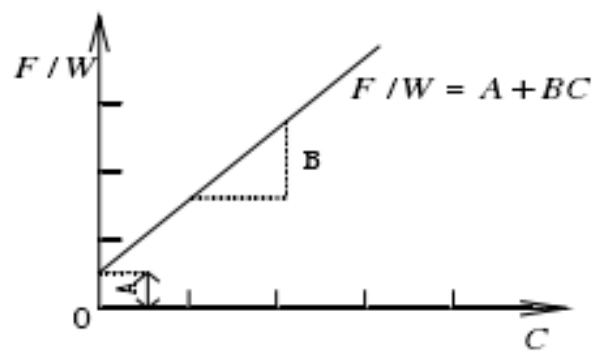

Figure 1: the relationship between the deformation and the collision force of the unit width According to the deformation of the automobile, the calculation of the energy losses is as follows:

$$
\begin{aligned}
& E=\int_{0}^{l}\left(A C+\frac{1}{2} B C^{2}+G\right) d W \\
& A=\frac{m b_{0} b_{1}}{g l} \\
& B=\frac{W b_{1}^{2}}{g l} \\
& G=\frac{W b_{0}^{2}}{2 g l}
\end{aligned}
$$

Among them, $\mathrm{m}$ is the weight of the vehicle; $\mathrm{W}$ is the width of deformation; 1 is the body width of the vehicle; $g$ is the acceleration of gravity. Therefore, refering to the energy theorem, the calculation of the collision speed is:

$$
\frac{m_{1} v_{10}^{2}}{2}+\frac{m_{2} v_{20}^{2}}{2}=\frac{m_{1} v_{1}^{2}}{2}+\frac{m_{2} v_{2}^{2}}{2}+\Delta E
$$

Among them, $m_{1}, m_{2}$ are the qualities of the twovehicles; $v_{10}, v_{20}, v_{1}, v_{2}$ stand for the two vehicles'speeds before and after the collision. $\Delta E$ is the energy lost in deformation during the collision.It can be seen from formula (4) and formula (5) that the speed before the collision can be calculated through the deformation of the vehicle, which is suitable for calculating the accident between the motorcycle head-on collision and the automobile, with no significant changes of automobile driving direction. But the difficulty is that it's not easy to use artificial measurement because of the depth of deformation in the traffic accident; selection of deformation stiffness coefficient is according to the stiffness coefficient based on more than 1000 real vehicle collision tests in the United States [11], in which there are differences between the actual stiffness coefficient of traffic accident, expanding the error in the calculation. In Formula (5), the speed of the motorcycle after the collision can be solved according to the braking traces of the automobile, the throw distance of the cyclist and the motorcycle, and the speed of the motorcycle before the collision can be solved according to the empirical formula. Foreign scholars have got the experience formula about the relationship between the motorcycle's deformation and the speed before collision through the experimental study on the motor vehicle frontal collision with the automobile [12]:

$$
v_{10}=\frac{1+m_{1} / m_{2}}{1+m_{1} / 1950}(1.5 D+12)
$$

$v_{10}$ is the speed of the motorcycle before collision, with the unit $\mathrm{km} / \mathrm{h} ; m_{1}$ is the quality of the motorcycle, whose unit is $\mathrm{kg} ; m_{2}$ is the quality of the automobile, with the unit $\mathrm{kg}$; $\mathrm{D}$ is the reduction of the motorcycle's wheel base, whose unit is $\mathrm{cm}$.

The effective collision speed formula is given in GA/T643-2006 Technical Appraisal Form Vehicle Speeds in the Typical Traffic Accidents:

$$
v_{e 1}=\frac{m_{2}\left(v_{10}-v_{20}\right)}{m_{1}+m_{2}} \quad v_{e 2}=\frac{m_{1}\left(v_{20}-v_{10}\right)}{m_{1}+m_{2}}
$$


$m_{1}, m_{2}$ are the qualities of the two vehicles; $v_{10}, v_{20}$ are the speeds of the two vehicles before collision.

The experience formula between the vehicle deformation and the effective collision speed can be obtained through real car collision test, the experience formula for effective collision speed of the car's front collision is:

$$
v_{e}=105.3 x
$$

$\mathrm{x}$ is the plastic deformation of the automobile.

The experience formula of the vehicle's effective speed in the rear-end collision is:

$$
v_{e}=35.8 m_{1} x /\left(m_{1}+m_{2}\right)+4.6
$$

The advantage lies in simple model and calculation stability according to the empirical formula of the deformation to solve the collision speed. However the disadvantage is the narrow range of using. Most of the experimental formula is obtained by experimental data fitting. when the the deformation of thev ehicle is too serious and the speed is faster than that of collision of the vehicle, the credibilit of the collision speed calculated by experimental formula is not high. In addition, the experimental formula has a certain scope. Different models, different forms of collision and accident environmental factors will have aneffect on the calculation results. If accurate collision speed is reconsrtucted, either the computer simulation technology must be used or the collision process must be deduced by using theoretical model, resulting in persuasive calculation.

\section{The uncertainty analysis of conservation of energy in the accident reconstruction}

The formula (6) expressed in the law of conservation of energy equation is:

$$
E=\sum_{i=1}^{n} E_{i}
$$

In the Equation, $\mathrm{E}$ is the total kinetic energy before the collision of the two vehicles; , $\mathrm{i}=1$, $2, \cdots, \mathrm{n}$ is the kinetic energy consumed in different forms of after the collision of the two vehicles.

There are three parts of the vehicles'energy consumption in in the process of an accident: the braking energy before collision, collision deformation energy, the energy transfer and the braking energy after collision. Different forms of consumption of energy is expressed in the equivalent velocity component (vi)of the vehicle as follows :

$$
\frac{1}{2} m_{2} v_{x o}^{2}=\frac{1}{2} m_{2}\left(v_{1}^{2}+v_{2}^{2}+v_{3}^{2}+\cdots+v_{n}^{2}\right)
$$

Namely:

$$
v_{\infty 0}=\sqrt{\sum_{i=1}^{n} v_{i}^{2}}
$$

In formula (11) the quivalent velocity component of energy consumed in braking can be calculated according to the braking traces:

$$
v_{j}=\sqrt{2 \varphi g S_{j}}+\frac{1}{2} \varphi g t \quad(\mathrm{j}=1,2, \cdots, \mathrm{m})
$$

In (11) the equivalent velocity component from deformation energy can be converted depending on the vehicle deformation $\mathrm{C}$ and the equivalent speed by using experiemental formula:

$$
v_{k}=f(C) \quad(\mathrm{k}=\mathrm{m}+1, \mathrm{~m}+2, \cdots, \mathrm{n})
$$

In the Equation, $\mathrm{f}$ is the relationship between the deformation and collision speed.

If the process of uncertainty evaluation is used to assess the method of accident reconstruction based on energy method, the evaluation model of uncertaintyis:

$$
v_{x}=f\left(v_{1}, v_{2}, \cdots, v_{i}\right)
$$

Calculate the uncertainty of the speed standard before collision, take the partial derivatives tively, on every parameter according to the formula to calculate the uncertainty of synthetic of $v_{20}$, namely: 


$$
u_{v_{1}}=\sqrt{\sum_{i=1}^{n}\left(\frac{\partial v_{0}}{\partial v_{i}}\right)^{2} u_{x_{i}}^{2}}
$$

Factor k should be included when uncertainty of Synthetic standard results are calculated (15). The expanded uncertainty is solved by using the expanded uncertainty formula.

\section{Conclusion}

The model of trace reconstruction in the accident and accident analysis methods have been presented in this paper. In the trace reconstruction model, the applicability of the vehicle dynamics model plays a key role in describing the vehicle kinematics and dynamics. In accident analysis method based on deformation energy, the deformation energy method is suitable for calculating the motorcycle head-on collision with the vehicle, and with no significant changes of automobile driving direction. Uncertain analysis has been carried out in theprocess of the accident reconstruction based on the energy method. The energy consumption of the automobile in the process of accident can be converted to equivalent speed component of the vehicle. The equivalent speed component of the vehicle is analyzed as uncertain factors.

\section{References}

[1] Tao Chen, Lang Wei, Biao Gong, etc.The Sensitivity Analysis of Parameter in Vehicle to Vehicle collision accident reconstuction system [J]. Journal of Automobile Engineering, 2012, 34 (9) : 771-776.

[2] Cliff W E, Montgomery D T. Validation of PC-Crash-A Momentum-Based Accident Reconstruction Program [C]. SAE Technical Paper 960885, 1996.

[3] Piotr Swider, Jan Unarski, Wojcieh Wach. SMASH Program for Car Accident Simulation [C]. SAE Technical Paper 2000-01-0848, 2000.

[4] Yi-bing Li, Yungang Chen, Wu Wei-dong. The Construction of CM collision model and test [J]. Journal of Tsinghua University (Natural Science Edition), 2011, 41 (11) : 98-101.

[5] Tao Chen, Lang Wei, Yan Gao The Review on Identification Technology Aided with Computers between Vehicles in the Collision Accidents [J]. Chinese Journal of Safety Science, 2010, 20 (4) : 38- 43.

[6] Mc Henry Brian G, Mc Henry Raymond R. HVOSM-87 [J]. SAE Technical Paper 880228, 1988.

[7] Day, T., Differences Between EDVDS and Phase 4 [J]. SAE Technical Paper 1999-01-0103, 1999.

[8] Terry D. Day, Sydney G. Roberts, Allen R. York. SIMON: a new vehicle simulation model for vehicle design and safety research [J]. SAE Technical Paper 2001-01-0503, 2001.

[9] Lang Wei, Hao-xue Liu. Introduction to Automotive Safety Technology [M]. Beijing: People's Traffic Press, 1999.

[10] Campbell, K. Energy Basis for Collision Severity [J]. SAE Technical Paper 740565, 1974

[11] Strother, C., Woolley, R., James, M., and Warner, C. Crush Energy in Accident Reconstruction[J]. SAE Technical Paper 860371, 1986.

GA/T643-2006. The law of the People's Republic of China on Public Security Industry Standards [S] The Ministry of Public Security of the People's Republic of China, 2006. 